\title{
Infektionskontrolle - der Weg zur Überwindung der Resistenzproblematik auf Intensivstationen?
}

\author{
Tobias Welte
}

$\mathbf{R}$

esistenzen von Antibiotika gegenüber Erregern der wesentlichen Intensivinfektionen nehmen weltweit Jahr für Jahr zu. Während die Bedrohung durch Methicillin-resistente Staphylokokken (MRSA) oder durch multiresistente Non-Fermenter (wie Pseudomonas oder Acinetbacter spp.) seit langem bekannt ist, zeigen sich in den letzten Jahren dramatische Resistenzanstiege bei Enterokokken (gegenüber Vancomycin) und Enterobakterien (gegenüber Beta-Laktamen, Carbapenemen und Fluorchinolonen). In einer Reihe von Untersuchungen konnte gezeigt werden, dass das Vorliegen eines multiresistenten Erregers zu einer zwei- bis vierfach erhöhten Sterblichkeit auf der Intensivstation führt. Multiresistente Erreger erhöhen - je nach Gesundheitssystem mehr oder minder ausgeprägt - zudem die Kosten der Behandlung erheblich.

Im Kontrast zu der dynamischen Entwicklung bei Resistenzen, die sich mit dem steigenden Alter und der vermehrten Ko-Morbidität der Patienten in Zukunft noch beschleunigen wird, ist die Entwicklung neuer Antibiotika praktisch zum Erliegen gekommen. Mit Daptomycin und Tigecyclin sind in den letzten beiden Jahren nur 2 Substanzen zugelassen worden, die jedoch nicht bei allen Arten schwerer Infektion wirksam sind und insbesondere keine Effektivität bei Pneumonien gezeigt haben. Gerade im gramnegativen Bereich sind in den nächsten Jahren keine neuen antimikrobiellen Substanzen zu erwarten. Mit der Verbesserung der Inhalationstechnologie werden eine Reihe altbekannter Medikamente wie Amikacin und Ciprofloxacin als additive Therapie bei pulmonalen Infekten getestet, dies reicht jedoch nicht aus, um dem Anstieg an resistenten Erregern zu begegnen.

Der Vermeidung von Resistenzentstehung kommt daher in den nächsten Jahren die wesentliche Bedeutung zur Verringerung von Morbidität und Letalität - und damit zur Verringerung von Kosten - in der Intensivmedizin zu.
Eine der wesentlichen Maßnahmen zur Vermeidung der Ausbreitung von Infektionserregern ist die Infektionskontrolle. Screeningprogramme mit frühzeitiger Erfassung von multiresistenten Erregern, frühzeitiger Isolation von kolonisierten Patienten und eine aggressive Eradikationspolitik (sogenannte „Surge and destroy“-Strategie) haben dafür gesorgt, dass die Raten multiresistenter Erreger, vor allem die MRSA Prävalenz in Ländern wie den Niederlanden, der Schweiz und in Skandinavien gering waren. Auch für Deutschland konnte gezeigt werden, dass Screeningverfahren, vor allem in Hochrisikobereichen wie Hämatologie/Onkologie, Neurologie/Neurochirurgie, Dialyse, Dermatologie und auf den Intensivstationen in Kombination mit sogenannten „Alert“-Systemen zu einer Reduktion von Resistenzraten beiträgt. Unter „Alert“-Systemen versteht man, dass ein Erreger, der einmal mit einem resistenten Erreger identifiziert wurde im Krankenhaussystem entsprechend gekennzeichnet ist, sodass bei Wiederaufnahme dessselben Patienten sofort entsprechende Untersuchungen und Maßnahmen eingeleitet werden können.

Screeningmaßnahmen sind, obwohl vordergründig teuer, kosteneffektiv, weil die Reduktion der Resistenzrate zu dramatischen Einsparungen der Behandlungskosten führt. Sie sind daher heute anerkannterweise als Maßnahmen der ersten Wahl zur Infektionskontrolle anzusehen.

In gleicher Weise gilt es inzwischen als erwiesen, dass den Händen eine herausragende Bedeutung in der Reduktion der Erregerübertragung zukommt. Das Bundesministerium für Gesundheit hat deshalb landesweit im letzten Jahr die „Aktion saubere Hände“ in deutschen Krankenhäusern unterstützt, um einheitlich Aufklärungs- und Schulungsmaßnahmen in Deutschland durchzuführen.

Andere Maßnahmen wie die Isolation von Patienten oder spezifische Regeln zur Reduktion der Rate spezieller Infektionen (z. B. die Oberkörperhochlagerung 
des Beatmeten zur Pneumonieprophylaxe) sind in ihrer Wertigkeit nicht sicher einzuschätzen und teilweise umstritten. Sie sind auch im Bezug auf ihre Wirkung stark von infrastrukturellen Gegebenheiten der Krankenhäuser abhängig. So kann der Aufwand, der für Isolierungsmaßnahmen von Patienten notwendig ist, bei Personalmangel zur Vernachlässigung anderer wesentlicher Maßnahmen wie der Händehygiene führen und ist dann eher kontraproduktiv. Bei guter Personalbesetzung und Zimmerpflege ist per se eine Einzelbetreuung von Patienten gegeben, dann erübrigt sich letztlich der Aufwand für die Isolation. Hinzu kommt, dass verschiedene multiresistente Erreger unterschiedliche Übertragungsrisiken aufweisen und daher im Hinblick auf die notwendigen Infektionskontrollmaßnahmen unterschiedlich zu handhaben sind. Eine an generellen Prinzipien orientierte, auf die lokale Situation zugeschnittene standardisierte und ausreichend überwachte Infektionskontrolle ist daher für jedes Krankenhaus zu empfehlen.

In den letzten Jahren ist die Zahl der Hygienefachkräfte und für Hygiene verantwortlichen Ärzte immer weiter reduziert worden. Dies spart vordergründig Ressourcen, ist jedoch aufgrund der hohen Kosten der Multiresistenz wenig kosteneffektiv. Ein Umdenken der Krankenhausträger verbunden mit - aufgrund der erheblichen langfristigen Relevanz von Resistenz für die Gesundheitssysteme und die Gesellschaft - klaren Vorgaben der Politik ist daher einzufordern.

Die Entstehung von Resistenz korreliert direkt mit dem Antibiotikaverbrauch sowohl im ambulanten wie im stationären Bereich. Der Reduktion des Antibiotikaverbrauchs ohne Gefährdung von Patienten kommt daher eine wesentliche Bedeutung für die Zukunft zu. Drei Dinge scheinen hierbei erfolgversprechend: Zum einen ist hier der Einsatz moderner Biomarker als Hilfskriterium bei der Entscheidung zur Antibiotikatherapie und zur Steuerung der Therapiedauer zu nennen. Im ambulanten Bereich und bei leichter kranken Patienten ist eine Antibiotikatherapie vielfach überflüssig, bei schwerer Kranken wird sie stets notwendig sein, in Deutschland wird jedoch in der Regel wesentlich zu lang therapiert. Resistenzentstehung korreliert jedoch direkt mit der Länge der Therapie. Zum anderen wissen wir heute, dass die schnelle, breit wirksame hochdosierte Antibiotikatherapie bei schwer kranken Patienten Überleben sichert. Der Therapiebeginn liegt dabei in der Regel vor der Identifikation des auslösenden Erregers. Deeskalationsstrategien (von Kombinations- auf Monotherapie und von breiter wirksamen auf gezielt eingesetzte Substanzen) werden immer wieder propa- giert, in der Praxis werden sie jedoch selten angewandt. Gezielte Deeskalation könnte jedoch erheblich den Selektionsdruck von Erregern reduzieren. Dies setzt jedoch - und das ist die dritte und wesentliche Strategie für die Zukunft - ein viel breiteres infektiologisches Wissen von Ärzten voraus. Standardisierte Diagnostik- und Therapieschemata und regelmäßige Schulung von Ärzten reduzieren den Antibiotikaverbrauch und damit die Resistenzentstehung, reduzieren Morbidität und Letalität und damit letztlich Kosten für das Gesundheitssystem.

Eines muss einem bei allen geschilderten Möglichkeiten zur Resistenzvermeidung klar sein. Hospitalinfektionen und Resistenzentstehung von Erregern können reduziert werden, vermeidbar sind sie nicht. Alter und Grundkrankheit von Patienten sind wesentliche Risikofaktoren für Resistenzentstehung, in einer älter werdenden, multimorbideren Gesellschaft werden diese Probleme immer bestehen bleiben. Reißerische Zeitungsberichte, selbst in eigentlich seriösen Zeitschriften wie dem „Spiegel“, die nahelegen, dass 60000 Todesfälle aufgrund nosokomialer Infektionen vermeidbar wären, wenn wir bessere Händehygiene betreiben, sind barer Unfug und verunsichern nur die Öffentlichkeit. Wir können uns verbessern, zur Überwindung des Resistenzproblems brauchen wir jedoch intensive Forschung und die Entwicklung neuer Therapieprinzipien.

J. J. Gould schreibt im „Book of the Worms“: „Mikroben waren lange vor uns da und werden lange nach uns weiter existieren. Sie betrachten unsere Anstrengungen, Sie zu überwinden, belustigt, denn sie wissen, dass sie siegen werden." Dem ist nichts hinzuzufügen.

\section{Korrespondenzadresse}

Prof. Dr. Tobias Welte

Medizinische Hochschule Hannover

Abteilung Pneumologie

Carl Neuberg Straße 1

30625 Hannover

E-Mail: Welte.Tobias@mh-hannover.de 